AMBIENT APPRAISAL

\title{
Cogitation on Hydrocarbon Contaminants in Shallow Groundwater around Kaduna Ref ining and Petrochemical Company, Nigeria
}

\author{
Murtala Shehu Ahmed', Adamu Idris Tanko \\ ${ }^{1}$ Kaduna Refining and Petrochemical Company Limited \\ (KRPC/NNPC), Kaduna, Nigeria \\ ${ }^{2}$ Earth and Environmental Sciences, Bayero University, Kano \\ Study Area: Kaduna, Nigeria \\ Coordinates: $7^{\circ} 28^{\prime} 45^{\prime \prime}-7^{\circ} 30^{\prime \prime} \mathrm{O}^{\prime \prime} \mathrm{E} ; 10^{\circ} 24^{\prime} \mathrm{O}^{\prime \prime}-10^{\circ}$ \\ $25{ }^{\prime} 20^{\prime \prime} \mathrm{N}$.
}

Key words: Benzene, Toluene, Ethylbenzene, Xylene, BTEX,

\section{Abstract}

Kaduna refinery is the third petroleum refinery in Nigeria which consists of the fuels, lubricants and petrochemical plants. An investigation was carried out on groundwater around the refinery to ascertain the presence of hydrocarbon contaminants. Twenty-six groundwater samples were collected in the dry and rainy seasons and analyzed for identification of aromatic compounds followed by the analysis of five selected samples for the individual compounds of benzene, toluene, ethylbenzene, and xylene (BTEX) determination. Only one sample out of the twenty-six indicated the presence of aromatic hydrocarbons. The sample was selected with four others for the GC-MS analysis. None of the samples confirmed the presence of BTEX in groundwater of the area. Benzene derivative compounds were however, found in one sample. Installation of properly designed monitoring wells around the refinery was recommended for continuous groundwater monitoring

smelling plant extracts. Aliphatic and aromatic hydrocarbons are likely the most frequent contaminants in the environment. They are part of different distillation products such as crude oils, gasoline, kerosene, diesel, heavy oils, or asphalts, and are used in a wide range of applications such as fuel or lubrication (Kralik et al., 2004). One of the main sources of hydrocarbon contamination of groundwater includes production and refining of crude petroleum and leaking of underground storage tanks (USTs) from the petrol stations. Most components of hydrocarbons are hazardous to both human life and the environment, some are either known or suspected human carcinogens (benzene), some have an adverse impact on the nervous system (toluene), while some are known to cause cardiovascular damage (ethylbenzene), they are thus, subject to strictest regulation.

When hydrocarbon compounds are released, they undergo physical, chemical and biological changes (Williams et al., 2006). Their degradations depend on the physical and chemical properties of the hydrocarbon. Once hydrocarbon enters into the soil, it migrates downward through the unsaturated zone under the influence of gravity (Abriola \& Pinder, 1985). Hydrocarbon may occur in soil in four different phases, dissolved in water, sorbed on solid particles, comprising the soil gas and due to their aromatic is derived from degradation of certain pleasant-

*Corresponding Author: almurtadha2003@yahoo.com 
limited solubility forming an individual liquid phase known as non-aqueous phase liquid (NAPL) (Konecny et al., 2003; Bear \& Cheng, 2009; Bear 1979) but it is more customarily encountered as a distinct no-aqueous phase (Abriola \& Pinder, 1985). In the capillary zone, the NAPL infiltration may be retained by the capillary forces leading to lateral migration, and the vapour may extend beyond the main area of contamination as a result of volatilization of light components. Some of the contaminants especially in large spills reach the water table and the soluble components dissolved, forming a plume of contaminated water extending outward beyond the contamination zone as part of the groundwater. The NAPL may move on its own pressure gradient within the capillary fringe zone and eventually depress natural groundwater levels (Abriola \& Pinder, 1985). Perhaps the NAPL an almost immiscible integrant in water, its small solubility is sufficient to cause groundwater contamination (Bear \& Cheng, 2009). Benzene, toluene, ethyl benzene and xylene (BTEX) are the most common aromatic compounds in petroleum and also the most soluble with a lowest organic carbon partition coefficient (Koc). Benzene is the most toxic and mobile in groundwater, it is the main groundwater contaminant of concern at petroleum release sites (Williams et al., 2006; Lovanh et al., 200o) and it is, therefore, subject to the strictest clean up standard and thus, dictates the need for remedial action in many places (Lovanh et al., 2000).

Petroleum refining process consists of a complex combination of interdependent operations which involves the separation of crude molecular constituents, molecular cracking, molecular rebuilding and solvent finishing (Department of Petroleum Resources (DPR, 2002). Petroleum hydrocarbons are of environmental concern due to their volatility, adverse effect through human and environmental toxicity, mobility from point of release (lighter ends), persistent in the environment (larger and branched chain), impairment of aesthetic value such as offensive odour, taste or appearance (Saskatchewan Ministry of Environment, 2008). In the processing of crude oil, several gaseous, liquid and solid hydrocarbon wastes are discharged into the environment which may find their ways into the atmosphere, surface water, soil, and groundwater. Contaminants may reach groundwater system due to localized spill incidence from piping, storage, processing, and loading of the products as well as poor handling of waste products.

Having discovered that the risk of groundwater contamination by hydrocarbons from the KRPC operations is at best, moderate (Ahmed, 2016), it is pertinent to investigate if the operations have impacted on the groundwater of the neighbouring areas. Several researches conducted in the neighbourhood of the refinery have indicated that, the surface water, soil, and groundwater were contaminated by heavy metals among other contaminants ( Amadi et al., 2014; Al-Amin 2013, Lekwot et al., 2012, Vivien et.al., 2012, Akpan et.al., 2008; Al-Amin, 2006). For obvious reason, this investigations focused on the hydrocarbon contaminants in the groundwater sources of the settlements located near the refinery. The settlements under review (Rido, Kpam, Railway quarters, and Chidunu), encircled the refinery and their choice reflected the radial pattern of groundwater flow in the area as well as proximity. Since it is an established fact that, the main contaminants of concern at a crude oil and petroleum products release site are benzene, toluene, ethylbenzene and xylene (BTEX) compounds, this research concentrated on ascertaining their presence in groundwater as a clear indication of hydrocarbon contamination from the refinery. BTEX in groundwater as opined by Fetter in Kralik et al (2004), is a diagnostic of the oil spill. Two analytical techniques, Fourier Transform Infrared Spectroscopy (FTIR) and Gas Chromatography/ Mass Spectrometry (GCMS) were used in the investigation.

\section{Methodology:}

Study area:Kaduna Refining and Petrochemical Company (KRPC) is located at the outskirt of Kaduna metropolis. It was commissioned in 1980 with an initial capacity of 100,00o barrel per stream day (bpsd) which was increased to 110,000 bpsd by 1985 . It was designed to process both Nigerian and imported crude oils into fuels and lubes products. A 30,ooo metric tonnes per annum Linear Alkyl Benzene (LAB) plant was also commissioned in December 1988 in the complex. KRPC produces petrol/gasoline (Premium Motor Spirit; PMS), Dual Purpose Kerosene (DPK), diesel or Automotive Gas Oil (AGO), asphalt, Linear Alkyl Benzene (LAB), base oils, Liquefied Petroleum Gas (LPG) and fuel oil.

Sources of contaminated water which pose a serious threat to groundwater system include sour water stripper condensate, cooling tower blowdown, caustic wash water, desalter water, oily process area stormwater, oily cleaning water, and spent chemicals (DPR, 2002). Among other contaminants, BTEX compounds (benzene, toluene, ethylbenzene, and xylene), and Polycyclic Aromatic Hydrocarbons (PAHs), as well as heavy metals characterized the effluent (DPR, 2002). Sources of solid waste in the refinery include sludge from crude and product tanks, corrosion products, solid from cleaning and maintenance operation, spent catalyst, refuse, sludge from wastewater treatment plant, empty metals/plastic chemical containers. Oily sludge, heavy metals, organic constituents, residue, and rust characterized the waste (DPR, 2002).

A treatment plant for wastewater was constructed in the refinery to treat all the effluents into the acceptable level before it is finally discharged into the River Romi. Equally, some of the solid waste is designed to be incinerated and the end products are used in making 
fertilizer. There is also an existing contractual agreement between the company and the Kaduna State Environmental Protection Agency (KEPA) to evacuate the solid wastes to a safe site.

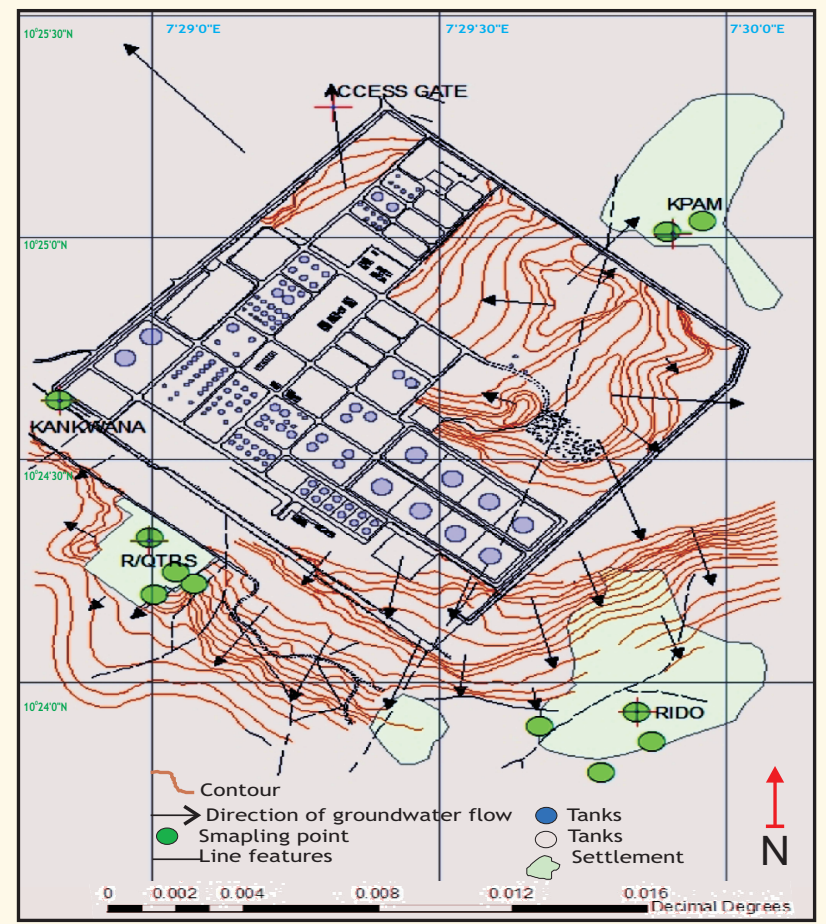

Figure 1: Groundwater Flow Direction Around Kaduna Refinery (Modified from Center for Energy Research and Training, 1991)

Groundwater Sampling: for hydrocarbon analysis in the laboratory groundwater samples were collected from the communities surrounding the KRPC (Figure-1). Monitoring wells were selected perpendicular to the groundwater flow directions to trap the possible hydrocarbon plume movement. A control well was selected far away from the refinery (Unguwan Dosa) and away from possible hydrocarbon sources to serve as a control sample. A total of thirteen monitoring wells were selected for screening, which were later reduced to five. Out of the thirteen wells, only two were boreholes, the remaining were open wells. Samples were collected in the dry (February, 2015) and wet seasons (August, 2014 \& August 2015) to detect any seasonal variation either due to recharge or rise in groundwater level. All samples were collected in sampling bottles prepared and obtained from the National Research Institute on Chemical Technology (NARICT) Zaria laboratory. The samples were stored in an ice-packed cooler to avoid the volatilization of hydrocarbon components due to temperature rise. Samples were immediately transferred to the laboratory for extraction and hydrocarbon analyses.

Laboratory Analysis : Hydrocarbon extractions were performed in the laboratory first before running the analysis. Analyses were conducted in the laboratory of NARICT, Zaria.
Determination of organic compound using Fourier Transform Infrared Spectroscopy (FTIR): the samples of about $1 \mathrm{ml}$ were absorbed and poured into a cell. This cell was then compressed into a thin pellet for $5 \mathrm{~min}$. The sample was then analyzed using Shimadzu 8300 spectrometer and the spectrum was recorded in a spectral range.

Determination of Hydrocarbons (BTEX) using Gas Chromatography-Mass Spectrometer (GC-MS): isolation and extractions were conducted using Liquid-Liquid extraction (LLE) methods. $20 \mathrm{ml}$ of an aqueous sample was placed in a 10oml beaker, petroleum-ether (20ml) extraction solvent which has good chromatographic behavior (good extraction properties for the compounds of interest ) was then injected rapidly into the solution by use of a 1.ooml gastight syringe (Hamilton, USA) and the mixture was shaken gently. A cloudy solution (water sample, Pet-ether) was formed in the test tube. The solution was then put in an oven at $45^{\circ} \mathrm{C}$ for about $30 \mathrm{~min}$. The mixture was then centrifuged using Centurion Scientific (UK) model $2010 \mathrm{D}$ for $2.0 \mathrm{~min}$ at $5,000 \mathrm{rpm}$, causing the dispersed fine droplets of the extraction phase to settle to the bottom of the tube; 0.50ml of the settled extraction phase was then collected using a $1.00 \mathrm{ml}$ microsyringe and injected for GC-FID analysis. Gas chromatography was performed with a Shimadzu GC 2010 instrument with splitless injection and a fame-ionization detector. Compounds were separated on a $9 \mathrm{~mm}$ capillary column coated with a $0.25 \mathrm{~mm}$ film of BP-5 (95\% methyl, $5 \%$ phenyl copolymer; SGE). Ultra-pure helium (99.9999\%), passed through a molecular sieve trap and an oxygen trap was used as carrier gas at a constant linear velocity of $30 \mathrm{~cm}^{\mathrm{s}-1}$. The injection port was held at high temperature and used in splitless mode with a splitless time $0.5 \mathrm{~min}$. The oven temperature was held at about $300^{\circ} \mathrm{C}$. The total time for one GC run was $15 \mathrm{~min}$.

\section{Results and Discussion:}

Fourier Transform Infrared Spectroscopy (FTIR) analysis: a preliminary investigation of aromatic compounds, the functional group which BTEX belongs was conducted by analyzing a total of twenty-six groundwater samples in the NARICT laboratory using the SHIMADZU FTIR-840oS Fourier Transform Infrared Spectrophotometer. The recorded peaks were compared with the correlation charts (Table-1) to deduce the functional groups of each absorption peak. Since the study was basically concern with the aromatic hydrocarbons, a diagnosis guide for aromatic according to Coates (2000) was strictly adhered to, to avoid assigning wrong interpretation due to an inherent overlap of the absorption frequency $\left(\mathrm{cm}^{-1}\right)$. Following the guideline, aromatics were found in only one sample tagged Railway quarters 2 which is located opposite the railway station behind the refinery. The sample which was collected in February 2015, recorded a peak at 
$1528.64 \mathrm{~cm}^{-1}$, ordinarily, it might be assigned to the amides group but the presence of two other peaks at $672.21 \mathrm{~cm}^{-1}$ and $779.27 \mathrm{~cm}^{-1}$ indicated the presence of aromatics as opined by Coates (2000). Other samples that recorded peaks at around $1533-156 \mathrm{ocm}^{-1}$ such as Railway quarters 3, Rido 2, Chidunu 1 \& 2 could not be supported by other peaks which are indicators of the presence of aromatics, for this reason, they were interpreted as amides. Table- 2 summarizes the result and interpretations of the analysis. From the analyses, it is evident that only one sample out of the total samples of 26 (for both wet and dry seasons), has aromatic compounds and thus, expected to contain the BTEX compounds. Such sample and four others were subjected to more robust GCMS analysis in order to unveil their real contents. Interestingly, the control sample collected at Unguwan Dosa, more than 20KM away from the refinery contains similar compounds (alkyl halides, amines, amides and the like) as obtained within the vicinity of the ref inery. This is possible as FTIR is capable of capturing both organic and inorganic compounds (Coates, 2000).

Gas Chromatography-Mass Spectrometry (GC-MS) analysis: to detect the presence of BTEX compound, five water samples from the neighborhood of Kaduna refinery were collected and subjected to GC-MS analysis. Again none of the samples was found to have the individual compounds of benzene, toluene, ethylbenzene, and xylenes. The samples were found to have different types of hydrocarbons especially the acids and esters among others (Table- 3). In the sample collected from Kankwana water well (behind the refinery's effluent discharge point tagged as Sample A), the majority of the hydrocarbon constituents (over $70 \%$ ) were acids of different types. Their carbon numbers ranged between $C_{11}$ and $C_{22}$. About $4 \%$ of the constituents were the esters $\left(C_{21}-C_{39}\right)$ of some of the acids such as octadecanoic, oleic, hexadecanoic among others. Alcohol and aldehydes account for up to $11 \%$ of the hydrocarbon content in the sample, they were mostly within the range of $\mathrm{C}_{11}-\mathrm{C}_{19}$. About $9 \%$ of the hydrocarbon content in the sample was oxacyclotetradecan-2-one with the remaining percentage (about $4 \%$ ) from relatively lower molecular compounds $\left(\mathrm{C}_{9}-\mathrm{C}_{10}\right)$ of fluorine derivatives. BTEX compounds were not found in the sample.

The sample collected from Kpam water well (Sample B) indicated the presence of hydrocarbons with more than $96 \%$ comprising different forms of carboxylic acids. Ester of octadecanoic acid account for the remaining $4 \%$. Again, the analysis failed to indicate the presence of BTEX in the sample.

About $87 \%$ of the hydrocarbon content in the sample collected from water well close to the tanker parking area of the depot (Sample-C) are also the carboxylic acid compounds with a carbon number between 11-22. Esters account for more than $5 \%$ in the sample while butane, 1, 3dichloro-2-methyl- and oxacyclotetradecane-2, 11-dione, 13-methyl- $\left(\mathrm{C}_{5}\right.$ and $\mathrm{C}_{14}$ respectively account for about $1 \%$ each. About $2 \%$ of the hydrocarbon content in the sample were benzene compounds derivatives: 2,6,2',6'Tetramethylazobenzene $\left(\mathrm{C}_{16} \mathrm{H}_{18} \mathrm{~N}_{2} \mathrm{O}_{2}\right)$, Benzaldehyde, 2hydroxy-4-methyl- $\left(\mathrm{C}_{8} \mathrm{H}_{8} \mathrm{O}_{2}\right)$, Benzenemethanol, 4-nitro$\left(\mathrm{C}_{7} \mathrm{H}_{7} \mathrm{NO}_{3}\right)$ and Benzene, [ (2, 2-dimethylcyclopropyl)methyl]- $\left(\mathrm{C}_{12} \mathrm{H}_{16}\right)$ although no actual benzene $\left(\mathrm{C}_{6}\right)$ was detected. Carboxylic acid compounds $\left(\mathrm{C}_{1-1-12}\right)$ constituted about $65 \%$ of the hydrocarbon content in the groundwater sample collected from the Railway quarters (Sample D). About 9\% each of the content were esters and aldehydes, $12 \%$ alcohols and $5 \%$ fluorine compounds. None of the BTEX compounds was detected.

Table 1: Characteristic IR Absorptions $\mathrm{m}=$ medium, $\mathrm{w}=$ weak, $\mathrm{s}=$ strong, $\mathrm{n}=$ narrow, $\mathrm{b}=$ broad, $\mathrm{sh}=$ sharp

\begin{tabular}{|c|c|c|}
\hline Frequency, $\mathrm{cm}^{-1}$ & Bond & Functional group \\
\hline $36403610(\mathrm{~s}, \mathrm{sh})$ & OH stretch, & free hydroxyl alcohols, phenols \\
\hline $35003200(\mathrm{~s}, \mathrm{~b})$ & $\mathrm{OH}$ stretch, & Hbonded alcohols, phenols \\
\hline $34003250(\mathrm{~m})$ & NH stretch & $1^{\circ}, 2^{\circ}$ amines, amides \\
\hline $33002500(\mathrm{~m})$ & OH stretch & carboxylic acids \\
\hline $33303270(\mathrm{n}, \mathrm{s})$ & $\mathrm{C} \equiv \mathrm{CH}: \mathrm{CH}$ str. & alkynes (terminal) \\
\hline $31003000(\mathrm{~s})$ & $\mathrm{CH}$ stretch & aromatics \\
\hline $31003000(\mathrm{~m})$ & $=\mathrm{CH}$ stretch & alkenes \\
\hline $30002850(\mathrm{~m})$ & $\mathrm{CH}$ stretch & alkanes \\
\hline $28302695(\mathrm{~m})$ & $\mathrm{HC}=\mathrm{O}: \mathrm{CH}$ str. & aldehydes \\
\hline $22602210(v)$ & $\mathrm{C}_{\equiv \mathrm{N}}$ stretch & nitriles \\
\hline $22602100(w)$ & $\mathrm{C} \equiv \mathrm{C}$ stretch & alkynes \\
\hline $17601665(\mathrm{~s})$ & $\overline{\mathrm{C}}=\mathrm{O}$ stretch & carbonyls (general) \\
\hline $17601690(\mathrm{~s})$ & $\mathrm{C}=\mathrm{O}$ stretch & carboxylic acids \\
\hline $17501735(\mathrm{~s})$ & $\mathrm{C}=\mathrm{O}$ stretch & esters, saturated aliphatic \\
\hline $17401720(\mathrm{~s})$ & $\mathrm{C}=\mathrm{O}$ stretch & aldehydes, saturated aliphatic \\
\hline $17301715(\mathrm{~s})$ & $C=\mathrm{O}$ str. $\alpha, \beta$ & unsaturated esters \\
\hline $1715(s)$ & $\mathrm{C}=\mathrm{O}$ stretch & ketones, saturated aliphatic \\
\hline $17101665(\mathrm{~s})$ & $C=\mathrm{O}$ str. $\alpha, \beta$ & unsaturated aldehydes,ketones \\
\hline $16801640(\mathrm{~m})$ & $\mathrm{C}=\mathrm{C}$ stretch & alkenes \\
\hline $16501580(\mathrm{~m})$ & $\mathrm{NH}$ bend $\mathrm{r}^{\circ}$ & amines \\
\hline $16001585(\mathrm{~m})$ & CC str. (inring) & aromatics \\
\hline 15501475 (s) & NO asym. str. & nitro compounds \\
\hline $15001400(\mathrm{~m})$ & CC str. (inring) & aromatics \\
\hline $14701450(\mathrm{~m})$ & $\mathrm{CH}$ bend & alkanes \\
\hline $13701350(\mathrm{~m})$ & $\mathrm{CH}$ rock & alkanes \\
\hline $13601290(\mathrm{~m})$ & NO sym. str. & nitro compounds \\
\hline $13351250(\mathrm{~s})$ & CN stretch & aromatic amines \\
\hline $13201000(\mathrm{~s})$ & CO stretch & $\begin{array}{l}\text { alcohols, carboxylic acids, } \\
\text { esters, ethers }\end{array}$ \\
\hline $13001150(\mathrm{~m})$ & $\mathrm{CH}$ wag $\left(\mathrm{CH}_{9} \mathrm{X}\right)$ & alkyl halides \\
\hline $12501020(\mathrm{~m})$ & CN stretch & aliphatic amines \\
\hline $1000650(\mathrm{~s})$ & $=\mathrm{CH}$ bend & alkenes \\
\hline $950910(\mathrm{~m})$ & OH bend & carboxylic acids \\
\hline $910665(\mathrm{~s}, \mathrm{~b})$ & $\mathrm{NH}$ wag $1^{\circ}, 2^{\circ}$ & amines \\
\hline $900675(\mathrm{~s})$ & CH "oop" & aromatics \\
\hline $850550(\mathrm{~m})$ & $\mathrm{CCl}$ stretch & alkyl halides \\
\hline $725720(\mathrm{~m})$ & $\mathrm{CH}$ rock & alkanes \\
\hline $700610(b, s)$ & $\mathrm{C} \equiv \mathrm{CH}: \mathrm{CH}$ & bendalkynes \\
\hline $690515(\mathrm{~m})$ & $\begin{array}{l}\mathrm{CBr} \text { stretch } \\
\text { str.=stretch; syr }\end{array}$ & $\begin{array}{l}\text { alkyl halides } \\
=\text { symmetric; asym=asymmetric }\end{array}$ \\
\hline
\end{tabular}

Hydrocarbon content in the groundwater sample 
collected from Rido revealed that about 90\% were from different acid compounds $\left(\mathrm{C}_{9}, \mathrm{C}_{14}-\mathrm{C}_{22}\right)$ with esters accounting for over $6 \%$. Other compounds present in the sample include alcohols, aldehydes among others. Again, none of the BTEX compounds was discovered in the sample.

Table-2: FTIR interpretation

\begin{tabular}{|c|c|c|}
\hline \multicolumn{3}{|c|}{ Table-2 a): RIDO 1 (Gidan Hassan) } \\
\hline \multirow{2}{*}{$\begin{array}{l}\text { August } 2014 \\
\text { Peak Interpretation }\end{array}$} & \multicolumn{2}{|c|}{ February 2015} \\
\hline & Peak & Interpretation \\
\hline 470.65 Alkyl halides & 401.21 & Alkyl halides \\
\hline 1273.06 Alkyl halides & $517 \cdot 9$ & Alkyl halides \\
\hline 1643.41 Amides/alkenes & 1016.52 & Alkyl halides \\
\hline 2088.98 (All four) $)^{* *}$ & 1640.51 & Amides \\
\hline \multirow[t]{3}{*}{3433.41 Amides } & 2061.01 & $(\text { All four })^{* *}$ \\
\hline & 2342.62 & \\
\hline & $\begin{array}{l}3444.02 \\
3850.04\end{array}$ & $\begin{array}{l}\text { Amides } \\
\text { Amines }\end{array}$ \\
\hline
\end{tabular}

\begin{tabular}{llll}
\hline \multicolumn{3}{c}{ Table 2b): RIDO 2 (Gidan Dagaci) } \\
August 2014 & February & \multicolumn{2}{c}{ 2015 } \\
\hline 455.22 & Alkyl halides & 406.3 & Alkyl halides \\
1642.44 & Amides/alkenes & 515.98 & Alkyl halides \\
2090.91 & (All four)** & 667.39 & Alkyl halides \\
3443.05 & Amides & 1038.7 & Alkyl halides \\
& & 1533.46 & Amides \\
& & 1641.48 & Amides/alkenes \\
& 2071.62 & (All four) \\
& & 3440.16 & Amides \\
\hline
\end{tabular}

\begin{tabular}{llll}
\hline \multicolumn{3}{c}{ Table 2c: RIDO 3 (Gidan Fati Maiwaina) } \\
August 2014 & February, 2015 \\
\hline Peak & Interpretation & Peak & Interpretation \\
476.3 & Alkyl halides & 409.89 & Alkyl halides \\
516.94 & Alkyl halides & 542.98 & Alkyl halides \\
1644.37 & Amides/alkenes & 1023.27 & Alkyl halides \\
2087.05 & (All four) ${ }^{* *}$ & 1183.37 & Alkyl halides \\
3347.57 & Amides/alcohols & 1455.34 & Alkanes \& alkyls \\
3430.51 & Amides & 1639.55 & Amides \\
3520.21 & Amines & 2061.01 & (All four) \\
& & 3445.94 & Amides \\
\hline
\end{tabular}

\begin{tabular}{llll}
\hline \multicolumn{3}{c}{ Table 2d: RIDO 4 (Mosque) } \\
August, 2014 & February, 2015 \\
\hline 479.33 & Alkyl halides & 419.53 & Alkyl halides \\
1272.1 & Alkyl halides & 501.51 & Alkyl halides \\
1643.41 & Amides/alkenes & 592.17 & Alkyl halides \\
2089.94 & (All four)** & 667.39 & Alkyl halides \\
3439.19 & Amides & 1159.26 & Alkyl halides \\
& & 1448.59 & \\
& & 1640.51 & Amides/alkenes \\
& 2065.83 & (All four)** \\
& 2947.33 & Alkanes \& alkyls/ \\
& & carboxylic acid \\
& & 3446.91 & Amides \\
\hline
\end{tabular}

\begin{tabular}{|c|c|c|c|}
\hline \multicolumn{4}{|c|}{ Table ze: Railway quarters 1} \\
\hline August & 2014 & February & 2015 \\
\hline 481.26 & Alkyl halides & 531.41 & Alkyl halides \\
\hline 1643.41 & Amides/alkenes & 1165.04 & Alkyl halides \\
\hline
\end{tabular}

2088.98 (All four) ** 3433.41 Amides

1639.55 Amides

2074.51 (All four)**

2346.48

3444.02 Amides

3862.58 Amides

\begin{tabular}{llll}
\hline \multicolumn{3}{c}{ Table 2f: Railway quarters 2} \\
August 2014 & February, 2015 \\
\hline 453.29 & Alkyl halides & 407.96 & Alkyl halides \\
1643.41 & Amides/alkenes & 510.19 & Alkyl halides \\
2090.91 & (All four) ${ }^{* *}$ & 672.21 & Aromatics \\
3427.62 & Amides & 779.27 & Aromatics \\
& & 1052.2 & Alkyl halides \\
& 1528.64 & Aromatics \\
& 1646.3 & Amides/alkenes \\
& 2033.04 & (All four) \\
& & 2947.33 & Alkanes \& alkyls \\
& 3443.05 & Amides \\
& 3721.77 & Amines
\end{tabular}

\begin{tabular}{|c|c|c|}
\hline \multicolumn{3}{|c|}{ Table 2g: Railway quarters 3} \\
\hline August, 2014 & \multicolumn{2}{|c|}{ February, 2015} \\
\hline 473.54 Alkyl halides & 404.1 & Alkyl halides \\
\hline 1643.41 Amides/alkenes & $544 \cdot 91$ & Alkyl halides \\
\hline 2089.94 (All four) $)^{* *}$ & $1037 \cdot 74$ & Alkyl halides \\
\hline 3441.12 Amides & 1161.19 & Alkyl halides \\
\hline & 1251.84 & Alkyl halides \\
\hline & 1542.14 & Amides \\
\hline & $1559 \cdot 5$ & Amides \\
\hline & 1642.44 & Amides/amines/alkenes \\
\hline & 2064.87 & $(\text { All four })^{* *}$ \\
\hline & 3444.02 & Amides \\
\hline
\end{tabular}

\begin{tabular}{llll}
\hline & \multicolumn{3}{l}{ Table 2h: } \\
Auguilway quarters 4 \\
\hline 501.51 & Alkyl halides & 384.81 & Alkyl halides \\
1642.44 & Amides/alkenes & 1026.16 & Alkyl halides \\
2088.98 & (All four) $^{* *}$ & 1639.55 & Amides \\
3318.64 & Amides & 2072.58 & (All four) \\
338* & Amides & 2336.84 & \\
3507.67 & Amines & 3444.02 & Amides \\
& & 3851.97 & Amines \\
\hline
\end{tabular}

\begin{tabular}{|c|c|c|c|}
\hline \multicolumn{4}{|c|}{ Table zi: kan-kwana } \\
\hline \multicolumn{2}{|c|}{ August, 2014} & \multicolumn{2}{|c|}{ February, 2015} \\
\hline 422.42 & Alkyl halides & 379.02 & Alkyl halides \\
\hline 457.14 & Alkyl halides & 1021.34 & Alkyl halides \\
\hline $1644 \cdot 37$ & Amides/alkenes & $1639 \cdot 55$ & Amides \\
\hline 2092.83 & $(\text { All four })^{* *}$ & 2070.65 & (All four $)^{* *}$ \\
\hline \multirow[t]{3}{*}{3446.91} & Amides & $2344 \cdot 55$ & \\
\hline & & 3444.02 & Amides \\
\hline & & 3854.87 & Amines \\
\hline
\end{tabular}

\begin{tabular}{llll}
\hline & & Table 2j: kpam 1 \\
August, 2014 & February, 2015 \\
\hline 496.69 & Alkyl halides & 415.67 & Alkyl halides \\
1644.37 & Amides/alkenes & 549.73 & Alkyl halides \\
2089.94 & (All four) & 1638.58 & Amides \\
3349.5 & Amides/alcohols & $\mathbf{2 0 6 2 . 9 4}$ & (All four) \\
3457.52 & Amides & 3414.12 & Amides \\
3558.78 & Amines & 3570.36 & Amides \\
& & & \\
& & &
\end{tabular}


Table $2 \mathrm{k}$ : kpam 2

\begin{tabular}{ll} 
August, 2014 & February, 2015 (no data) \\
\hline 440.75 & Alkyl halides \\
1644.37 & Amides/alkenes \\
2088.01 & (All four)** \\
3443.05 & Amides \\
\hline
\end{tabular}

\begin{tabular}{llll}
\hline \multicolumn{3}{c}{ Table 2 l: chidunu 1} \\
August, 2014 & February, 2015 \\
\hline 557.45 & Alkyl halides & 405.06 & Alkyl halides \\
1374.33 & Alkanes \& alkyls & 650.03 & Alkyl halides \\
1643.41 & Amides/alkenes & 1189.15 & Alkyl halides \\
2094.76 & (All four) & 1538.28 & Amides \\
2388.92 & & 1640.51 & Amides \\
3468.13 & Amides & 2063.9 & (All four) \\
3851.97 & Amines & 3438.23 & Amides \\
\hline
\end{tabular}

Table 2m: Chidunu 2

Chidunu 2- Gidan Ayuba (Aug '14)February, 2015

\begin{tabular}{llll}
\hline 497.65 & Alkyl halides & 390.6 & Alkyl halides \\
1254.74 & Alkyl halides & 468.72 & Alkyl halides \\
1641.48 & Amides/alkenes & 654.85 & Alkyl halides \\
2088.01 & (All four)** & 1539.25 & Amides \\
3444.02 & Amides & 1639.25 & Amides \\
& & 2071.62 & (All four) $^{* *}$ \\
& & 3440.16 & Amides \\
\hline
\end{tabular}

\begin{tabular}{llrl}
\hline \multicolumn{3}{c}{$\begin{array}{r}\text { Table 2n: Unguwan Dosa } \\
\text { February, 2015 }\end{array}$} \\
\hline 339.48 & Alkyl halides & 1031.95 & Alkyl halides \\
$\mathbf{1 4 5 4 . 3 8}$ & Alkenes \& alkyls & 1638.58 & Amines/amines \\
$\mathbf{2 0 7 4 . 5 1}$ & (All four) $^{* *}$ & 3443.05 & Amides \\
3854.87 & Amines & & \\
\hline
\end{tabular}

$(\text { All four })^{* *}=$ Allenes, ketenes, isocyanates, isothiocyanates

Table 3: Results of gc-ms analysis

Table za: Kankwana (Sample A)

\begin{tabular}{lll}
\hline Compound & $\mathrm{Mol}_{2}$ For. & $\%$ \\
\hline Undecanoic acid & $\mathrm{C}_{11} \mathrm{H}_{22} \mathrm{O}_{2}$ & 0.658 \\
Dodecanoic acid & $\mathrm{C}_{12} \mathrm{H}_{22} \mathrm{O}_{2}$ & 0.658 \\
Hexadecanoic acid & $\mathrm{C}_{16} \mathrm{H}_{32} \mathrm{O}_{2}$ & 9.14 \\
Tetradecanoic acid & $\mathrm{C}_{14} \mathrm{H}_{28} \mathrm{O}_{2}$ & $5 \cdot 372$ \\
Pentadecanoic & $\mathrm{C}_{15} \mathrm{H}_{30} \mathrm{O}_{2}$ & $5 \cdot 372$ \\
Octadecanoic acid & $\mathrm{C}_{18} \mathrm{H}_{36} \mathrm{O}_{2}$ & 6.288 \\
Heptadecanoic acid & $\mathrm{C}_{17} \mathrm{H}_{34} \mathrm{O}_{2}$ & 2.52 \\
Oleic Acid & $\mathrm{C}_{18} \mathrm{H}_{34} \mathrm{O}_{2}$ & 9.112 \\
Erucic acid & $\mathrm{C}_{22} \mathrm{H}_{42} \mathrm{O}_{2}$ & 9.112 \\
Hexadecenoic acid, & $\mathrm{C}_{16} \mathrm{H}_{30} \mathrm{O}_{2}$ & 18.224 \\
n-Hexadecanoic acid & $\mathrm{C}_{16} \mathrm{H}_{32} \mathrm{O}_{2}$ & 3.148 \\
(E)-13-Docosenoic acid & $\mathrm{C}_{22} \mathrm{H}_{42} \mathrm{O}_{2}$ & 0.378 \\
9-Octadecenoic acid (Z)-, & $\mathrm{C}_{21} \mathrm{H}_{40} \mathrm{O}_{4}$ & 0.378 \\
Octadecanoic acid, 2-(2-hydroxyethoxy) & & \\
ethyl ester & $\mathrm{C}_{22} \mathrm{H}_{44} \mathrm{O}_{4}$ & 1.574 \\
Oleic acid, 3-hydroxypropyl ester & $\mathrm{C}_{21} \mathrm{H}_{40} \mathrm{O}_{3}$ & 0.378 \\
Oxacyclotetradecan-2-one & $\mathrm{C}_{14} \mathrm{H}_{26} \mathrm{O}_{2}$ & 9.112 \\
Hexadecanoic acid, 1-[[[(2 aminoethoxy)hydro- & & \\
xyphosphinyl] oxy]methyl]-1,2-ethanediyl ester & $\mathrm{C}_{37} \mathrm{H}_{74} \mathrm{NO}_{8} \mathrm{P}$ & 1.432 \\
Hexadecanoic acid, 1-(hydroxymethyl)-1,2- & & \\
ethanediyl ester & $\mathrm{C}_{35} \mathrm{H}_{68} \mathrm{O}_{5}$ & 1.432 \\
& &
\end{tabular}

Octadecanoic acid, 2-hydroxy-1,3-

propanediyl ester

9-Octadecenal

10-Undecenal

9-Hexadecenal

9-Tetradecenal

11-Tridecen-1-ol

2-Octadecadecen-1-ol

12-Methyl-E,E-2,13-octadecadien-1-ol

1-Fluorodecane

1-Fluorononane

1-Propoxyheptane

$\mathrm{C}_{39} \mathrm{H}_{76} \mathrm{O}_{5} \quad 0.716$

$\mathrm{C}_{18} \mathrm{H}_{34} \mathrm{O} \quad 2.032$

$\mathrm{C}_{11} \mathrm{H}_{20} \mathrm{O} \quad 2.032$

$\mathrm{C}_{16} \mathrm{H}_{30} \mathrm{O} \quad 0.378$

$\mathrm{C}_{14} \mathrm{H}_{26} \mathrm{O} \quad 2.032$

$\mathrm{C}_{13} \mathrm{H}_{26} \mathrm{O} \quad 2.032$

$\mathrm{C}_{18} \mathrm{H}_{36} \mathrm{O} \quad 2.032$

$\mathrm{C}_{19} \mathrm{H}_{36} \mathrm{O} \quad 0.378$

$\mathrm{C}_{10} \mathrm{H}_{21} \mathrm{~F} \quad 1.628$

$\mathrm{C}_{9} \mathrm{H}_{19} \mathrm{~F} \quad 1.628$

Table zb: Kpam (Sample B)

Compound Mol. For. \%

Eicosanoic acid

Nonanoic acid

n-Hexadecanoic acid

Octadecanoic acid

Tetradecanoic acid

Oleic Acid

Erucic acid

E-9-Tetradecenoic acid

$\mathrm{C}_{10} \mathrm{H}_{22} \mathrm{O} \quad 0.814$

Hexadecenoic acid, Z-11-

9-Hexadecenoic acid

Heptadecanoic acid

Octadecanoic acid, 2-(2-hydroxyethoxy)

ethyl ester

$\mathrm{C}_{20} \mathrm{H}_{40} \mathrm{O}_{2} \quad 7.662$

$\mathrm{C}_{9} \mathrm{H}_{18} \mathrm{O}_{2} \quad 0.506$

$\mathrm{C}_{16} \mathrm{H}_{32} \mathrm{O}_{2} \quad 17.996$

$\mathrm{C}_{18} \mathrm{H}_{36} \mathrm{O}_{2} \quad 16.732$

$\mathrm{C}_{14} \mathrm{H}_{28} \mathrm{O}_{2} \quad 7.662$

$\mathrm{C}_{18} \mathrm{H}_{34} \mathrm{O}_{2} \quad 8.608$

$\mathrm{C}_{22} \mathrm{H}_{42} \mathrm{O}_{2} \quad 8.608$

$\mathrm{C}_{14} \mathrm{H}_{26} \mathrm{O}_{2} \quad 8.608$

$\mathrm{C}_{16} \mathrm{H}_{30} \mathrm{O}_{2} \quad 8.608$

$\mathrm{C}_{16} \mathrm{H}_{30} \mathrm{O}_{2} \quad 8.608$

$\mathrm{C}_{17} \mathrm{H}_{34} \mathrm{O}_{2} \quad 2.672$

\begin{tabular}{ccc}
$\mathrm{C}_{22} \mathrm{H}_{44} \mathrm{O}_{4}$ & 3.73 \\
\hline $\begin{array}{c}\text { Table 3c: Depot (Trailer parking area (Sample C) } \\
\text { Mol. For }\end{array}$ & $\%$ \\
\hline Compound & $\mathrm{C}_{9} \mathrm{H}_{10} \mathrm{O}_{2}$ & 0.328
\end{tabular}

6-Methyl-3-phenethylsulfanyl-[1,2,4]

triazin-5-ol

$\mathrm{C}_{12} \mathrm{H}_{13} \mathrm{~N}_{3} \mathrm{OS} 1.02$

cisZ-11,12-Epoxytetradecan-1-ol

6,11-Dimethyl-2,6,10-dodecatrien-1-ol

$\mathrm{C}_{14} \mathrm{H}_{28} \mathrm{O}_{2} \quad 0.26$

$\mathrm{C}_{14} \mathrm{H}_{24} \mathrm{O} \quad 0.156$

2,7-Dioxa-tricyclo[4-4.o.o(3,8)]deca-4,9-diene $\mathrm{C}_{8} \mathrm{H}_{8} \mathrm{O}_{2} \quad 0.328$

Farnesol isomer a $\quad \mathrm{C}_{15} \mathrm{H}_{26} \mathrm{O} \quad 0.156$

2,6,2',6'-Tetramethylazobenzene

Benzaldehyde, 2-hydroxy-4-methyl-

Benzenemethanol, 4-nitro-

Benzene,[(2,2-dimethylcyclopropyl)methyl]

Oxacyclododecan-2-one

Cyclopropanecarboxylic acid, benzyl ester

Octadecanoic acid, 2-(2-hydroxyethoxy)

ethyl ester

Butane, 1,3-dichloro-2-methyl-

$\mathrm{C}_{16} \mathrm{H}_{18} \mathrm{~N}_{2} \mathrm{O}_{2} \quad 0.328$

$\mathrm{C}_{8} \mathrm{H}_{8} \mathrm{O}_{2} \quad 0.328$

$\mathrm{C}_{7} \mathrm{H}_{7} \mathrm{NO}_{3} \quad 0.328$

$\mathrm{C}_{12} \mathrm{H}_{16} \quad 1.02$

$\mathrm{C}_{11} \mathrm{H}_{20} \mathrm{O}_{2} \quad 0.982$

$\mathrm{C}_{11} \mathrm{H}_{12} \mathrm{O}_{2} \quad 2.04$

Oxacyclotetradecane-2,11-dione, 13-methyl-

Undecanoic acid

Tetradecanoic acid

$\mathrm{C}_{22} \mathrm{H}_{44} \mathrm{O}_{4} \quad 3.03$

$\mathrm{C}_{5} \mathrm{H}_{10} \mathrm{Cl}_{2} \quad 1.02$

$\mathrm{C}_{14} \mathrm{H}_{24} \mathrm{O}_{3} \quad 0.982$

$\mathrm{C}_{11} \mathrm{H}_{22} \mathrm{O}_{2} \quad 0.874$

$\mathrm{C}_{14} \mathrm{H}_{28} \mathrm{O}_{2} \quad 2.23$

n-Hexadecanoic acid

$\mathrm{C}_{16} \mathrm{H}_{32} \mathrm{O}_{2} \quad 16.812$

Dodecanoic acid

Pentadecanoic acid

Octadecanoic acid

Oleic Acid

9-Hexadecenoic acid

Z-11-Tetradecenoic acid

$\mathrm{C}_{12} \mathrm{H}_{24} \mathrm{O}_{2} \quad 0.874$

$\mathrm{C}_{15} \mathrm{H}_{30} \mathrm{O}_{2} \quad 7.516$

$\mathrm{C}_{18} \mathrm{H}_{36} \mathrm{O}_{2} \quad 13.004$

$\mathrm{C}_{18} \mathrm{H}_{34} \mathrm{O}_{2} \quad 7.39$

$\mathrm{C}_{16} \mathrm{H}_{30} \mathrm{O}_{2} \quad 7.39$

$\mathrm{C}_{14} \mathrm{H}_{26} \mathrm{O}_{2} \quad 0.982$

Heptadecanoic acid

$\mathrm{C}_{17} \mathrm{H}_{34} \mathrm{O}_{2} \quad 8.994$ 
Erucic acid

Hexadecenoic acid, Z-11-

E-9-Tetradecenoic acid

9-Octadecynoic acid

Undec-10-ynoic acid

Eicosanoic acid

1,5,9-Undecatriene, 2,6,10-trimethyl-, (Z)-

Bicyclo[3.1.1] heptane, 2,6,6-trimethyl-, [1R-

(1.alpha.,2.beta.,5.alpha.)]-

Squalene

4,4,8-Trimethyl-non-5-enal
9,12-Octadecadienoic acid (Z,Z)-

$\begin{array}{ll}\mathrm{C}_{22} \mathrm{H}_{42} \mathrm{O}_{2} & 6.408 \\ \mathrm{C}_{16} \mathrm{H}_{30} \mathrm{O}_{2} & 7 \cdot 342 \\ \mathrm{C}_{14} \mathrm{H}_{26} \mathrm{O}_{2} & 5.474 \\ \mathrm{C}_{18} \mathrm{H}_{32} \mathrm{O}_{2} & 0.26 \\ \mathrm{C}_{18} \mathrm{H}_{32} \mathrm{O}_{2} & 0.26 \\ \mathrm{C}_{11} \mathrm{H}_{18} \mathrm{O}_{2} & 0.26 \\ \mathrm{C}_{20} \mathrm{H}_{40} \mathrm{O}_{2} & 0.906 \\ \mathrm{C}_{14} \mathrm{H}_{247} & 0.156 \\ \mathrm{C}_{10} \mathrm{H}_{18} & 0.26 \\ \mathrm{C}_{30} \mathrm{H}_{50} & 0.156 \\ \mathrm{C}_{12} \mathrm{H}_{22} \mathrm{O} & 0.156\end{array}$

Table 3d: Railway Quarters (Sample D)

Compound

Undecanoic acid

Dodecanoic acid

Tetradecanoic acid

n-Hexadecanoic acid

Pentadecanoic acid

Octadecanoic acid

Heptadecanoic acid

Oleic Acid

Erucic acid

Hexadecenoic acid, Z-11-

9-Hexadecenoic acid

E-9-Tetradecenoic acid

n-Hexadecanoic acid

(E)-13-Docosenoic acid

Decane, 1-fluoro-

1-Fluorononane

Hexadecanoic acid, 2,3-dihydroxypropyl

ester, (.+/-.)-

Pentafluoropropionic acid, tridecyl ester

Hexadecanoic acid, 1-[[[(2-aminoethoxy)hydroxy-

phosphinyl]oxy]methyl]-1,2-ethanediyl ester $\mathrm{C}_{37} \mathrm{H}_{74} \mathrm{NO}_{8} \mathrm{P} 1.57$

Octadecanoic acid, 2-(2-hydroxyethoxy)

ethyl ester

Hexadecanoic acid, 2,3-dihydroxypropyl ester

Octadecanoic acid, 2-hydroxy-1,3-

propanediyl ester

Dodecanoyl chloride

Decanoyl chloride

2-Methyl-Z,Z-3,13-octadecadienol

9-Octadecenal

10-Undecenal

E-9-Tetradecenal

2-Tridecenal, (E)-

2-Dodecenal, (E)-

3,11-Tetradecadien-1-ol

E-2-Octadecadecen-1-ol

3,11-Tetradecadien-1-ol

\begin{tabular}{lll}
\hline & $\mathrm{C}_{14} \mathrm{H}_{26} \mathrm{O}$ & 3.79 \\
\hline Compound & Table ze: Rido (Sample E) & \\
& Mol. For. & $\%$ \\
\hline 1-Decene & $\mathrm{C}_{10} \mathrm{H}_{20}$ & $\mathbf{0 . 2 2 2}$ \\
1-Undecene & $\mathrm{C}_{11} \mathrm{H}_{22}$ & $\mathbf{0 . 2 2 2}$ \\
3-Decene & $\mathrm{C}_{10} \mathrm{H}_{20}$ & $\mathbf{0 . 2 2 2}$
\end{tabular}

\begin{tabular}{|c|c|c|}
\hline 1-Dodecene & $\mathrm{C}_{12} \mathrm{H}_{24}$ & 0.222 \\
\hline 1,6,10-Dodecatrien-3-ol, 3,7,11-trimethyl-, & $\mathrm{C}_{15} \mathrm{H}_{26} \mathrm{O}$ & 0.158 \\
\hline -Thiabicyclo[4.1.o]heptane, 1-methyl- & $\mathrm{C}_{7} \mathrm{H}_{12} \mathrm{~S}$ & 0.21 \\
\hline $\begin{array}{l}\text { yclopropane, 1-(1'-propenyl)-2- } \\
\text { ydroxymethyl- }\end{array}$ & $\mathrm{C}_{7} \mathrm{H}_{12} \mathrm{O}$ & 0.21 \\
\hline -Oxabicyclo[2.2.1] heptan-3-one, 1,7,7-trimethyl & $\mathrm{C}_{9} \mathrm{H}_{12} \mathrm{O}_{2}$ & 0.21 \\
\hline Heptanol, 6-methyl- & $\mathrm{C}_{8} \mathrm{H}_{18} \mathrm{O}$ & 0.416 \\
\hline -Hexadecenoic acid & $\mathrm{C}_{16} \mathrm{H}_{30} \mathrm{O}_{2}$ & 9.012 \\
\hline Hexadecenoic acid, Z-11- & $\mathrm{C}_{16} \mathrm{H}_{30} \mathrm{O}_{2}$ & 18.024 \\
\hline enta & $\mathrm{C}_{15} \mathrm{H}_{30} \mathrm{O}_{2}$ & 5.216 \\
\hline Tetra & $\mathrm{C}_{14} \mathrm{H}_{28} \mathrm{O}_{2}$ & 9.822 \\
\hline Detade & $\mathrm{C}_{18} \mathrm{H}_{36} \mathrm{O}_{2}$ & 9.822 \\
\hline - $-\mathrm{He}$ & $\mathrm{C}_{16} \mathrm{H}_{32} \mathrm{O}_{2}$ & 14.918 \\
\hline canoic acid & $\mathrm{C}_{17} \mathrm{H}_{34} \mathrm{O}_{2}$ & $4 \cdot 726$ \\
\hline -methyl-oct-3-enoic acid & $\mathrm{C}_{9} \mathrm{H}_{16} \mathrm{O}_{3}$ & 0.21 \\
\hline Dleic & $\mathrm{C}_{18} \mathrm{H}_{34} \mathrm{O}_{2}$ & 9.012 \\
\hline Eruci & $\mathrm{C}_{22} \mathrm{H}_{42} \mathrm{O}_{2}$ & 9.012 \\
\hline loro-, methyl ester & $\mathrm{C}_{5} \mathrm{H}_{9} \mathrm{ClO}_{2}$ & 0.48 \\
\hline thyl-, methyl ester & $\mathrm{C}_{8} \mathrm{H}_{16} \mathrm{O}_{2}$ & 0.16 \\
\hline id, methyl ester & $\mathrm{C}_{10} \mathrm{H}_{18} \mathrm{O}_{2}$ & 0.37 \\
\hline acid, 2-(2-hydroxyethoxy) & $\mathrm{C}_{22} \mathrm{H}_{44} \mathrm{O}_{4}$ & 4.606 \\
\hline id, 2-pentadecyl ester & $\mathrm{C}_{20} \mathrm{H}_{40} \mathrm{O}_{2}$ & 0.416 \\
\hline $4-\operatorname{Tr}$ & $\mathrm{C}_{13} \mathrm{H}_{26}$ & 0.416 \\
\hline l-Non & $\mathrm{C}_{9} \mathrm{H}_{20} \mathrm{O}$ & 0.222 \\
\hline Isooc & $\mathrm{C}_{8} \mathrm{H}_{18} \mathrm{O}$ & 0.416 \\
\hline 2-Undecenal & $\mathrm{C}_{11} \mathrm{H}_{20} \mathrm{O}$ & 0.416 \\
\hline $\begin{array}{l}\text {,1-Cyclopropanedicarbonitrile, 2-methyl-2- } \\
\text { pentyl- }\end{array}$ & $\mathrm{C}_{11} \mathrm{H}_{16} \mathrm{~N}_{2}$ & 0.158 \\
\hline -Oxabicyclo[6.1.o]nonane, 1-methyl-, cis- & $\mathrm{C}_{9} \mathrm{H}_{16} \mathrm{O}$ & 0.158 \\
\hline Decen-2-one, 9-methyl-5-methylene- & $\mathrm{C}_{12} \mathrm{H}_{20} \mathrm{O}$ & 0.158 \\
\hline -Ethyl-6-heptafluorobutyryloxyoctane & $\mathrm{C}_{14} \mathrm{H}_{21} \mathrm{~F}_{7} \mathrm{O}_{2}$ & 0.158 \\
\hline
\end{tabular}

Mol.For $=$ Molecular formula

\section{Conclusion:}

Although the risk from the refinery appears to be moderate, traces of benzene compounds $\left(\mathrm{C}_{7}, \mathrm{C}_{8}, \mathrm{C}_{12}\right.$, and $\mathrm{C}_{16}$ ) were found in the groundwater sample collected around the refinery's access gate by trailer parking area (Sample C) in the GC-MS analysis. Also, the presence of aromatic compounds was discovered in one of the groundwater samples collected at railway quarters in the FTIR analysis. The two samples were both taken from open wells with poor casings and cover. Facts deduced from these occurrences are, either, the concentrations of the BTEX compounds especially benzene were higher in the groundwater than the observed content, or the contaminants were introduced via other pathways or both. Considering the volatility of the compounds, it could be stated that, most of the lighter hydrocarbon components especially benzene $\left(C_{6}\right)$ have volatilized into the air leaving the heavier ones in the groundwater as observed in Sample C. The result could be different if the samples were from properly designed monitoring wells. It is therefore evident that only a properly designed monitoring well network can reveal the extent of hydrocarbon content in the groundwater system around the refinery. It is 
recommended that properly designed monitoring wells surrounding the refinery should be drilled for continuous groundwater monitoring. Completion of the sludge pit remediation program in the ref inery and rehabilitation of the wastewater treatment plant is highly recommended. On the part of the communities, proper casing and cover of the water wells to avoid hydrocarbon contaminants influx should be strictly adhered to. This practice will help safeguard the safety of groundwater in the area.

\section{References:}

Abriola, L.M. \& Pinder, G.F. (1985): A multiphase approach to the modelling of porous media contamination by organic compounds, 1. Equation Development, Water Resour. Res., 21 (1):11-18.

Ahmed, M.S. (2016): Assessment of Groundwater Vulnerability and Risk of Hydrocarbon Contamination in Kaduna Metropolis, Nigeria, $\mathrm{PhD}$ thesis, Department of Geography, Bayero University, Kano.

Akpan, U.G., Afolabi, E.A. \& Okemini, K. (2008): Modeling and simulation of the effect of effluent from Kaduna refinery and petrochemical company on River Kaduna. AU J.T. (Technical report), 12(2):98-106.

Al-Amin, M.A. (2006): Environmental Impact Assessment of Kaduna Refinery on the Rido Region of Kaduna Metropolis, Ph.D. Thesis, Department of Geography, Ahmadu Bello University, Zaria.

Al-Amin, M.A. (2013): Energy production and environmental concerns in Nigeria: the case of Kaduna petroleum refinery on its host communities. J. Energy Tech. Policies, 3(10)69-77.

Amadi, A.N., Okoye, N.O., Alabi, A.D., Tukur, A. \& Angwa, E.M. (2014): Quality assessment of soil and groundwater near Kaduna refinery and petrochemical company, Northwest Nigeria.J.Sci. Res. Rep., 3(6):884-893.

Asadi, S.S., Vuppala, Ps., \& Reddy, M.A. (2007): Remote sensing and GIS techniques for evaluation of groundwater quality in municipal corporation of Hyderabad(Zone-V), India. Int. J. Environ. Res. Pub. Health, 4(1):45-52..

Bear, J. \& Cheng, A.H-D. (2009): Modelling groundwater flow and contaminant transport Pub. by: Springer Netherlands. XXI, 834 p.

Berg, M., Tran, H.C., Nguyen, T.C., Pham, H.V., Schertenleib, R., \& Giger, W. (2001) Arsenic contamination of groundwater and drinking water in Vietnam: a human health threat, Environ. Sci. Tech., 35(13):2621-2626.

Castillejos, K.M. (2010): Assessing integrated watershed management and spatial groundwater vulnerability to pollution in priority watersheds of the Yacyreta dam in Paraguay. Ph.D. dissertation, University of Wales (Bangor University) and Tropical Agricultural Research and Higher Education Center(CATIE).

Department of Petroleum Resources (2002) Environmental Guidelines and Standards for the Petroleum Industry in Nigeria (EGASPIN). Pub. by:The Department of Petroleum Resources, Nigeria.

Foster, S.S.D. \& Chilton, P.J. (2003): Groundwter: the processes and global significance of aquifer degradation. Philos.Trans. $R$. Soc. Lond. B. Biol. Sci., 358(1440):1957-1972.

Foster, S., Hirata, R., Gomes, D., D'Elia, M., \& Paris, M. (2002): Groundwater Quality Protection, a Guide for Water Utilities, Municipal Authorities, and Environment
Agencies. Pub. by: Groundwater Management Advisory Team (GW.MATE) in association with the Global Water Partnership, WHO-PAHO-CEPIS and UNESCO-ROSTLACPHI, World Bank, Washington, D.C.

Galadima, A., Garba, Z.N., Leke, L., Almustapha, M.N. \& Adam, I.K.(2011): Domestic water pollution among local communities in Nigeria- Causes and consequences, Eur. J. Sci. Res., 52(4):592-603.

Glasser, S., Gauthier-Warinner, J., Gurrieri, J. and Keely, J., Tucci, P., Summers, P., Wireman, M. and Mc Cormack, K.(2007) Technical Guide To Managing Groundwater Resources. Pub. by: United States Department of Agriculture (USDA), FS-881. 295p.

Konecny, F., Bohacek, Z., Muller, P., Kovarova, M. \& Sedlackova, I. (2003): Contamination of soils and groundwater by petroleum hydrocarbons and volatile organic compoundscase study: ELSLAV BRNO. Bull. Geosci., 78(3):225-239.

Kralik, M., Kranjc, A. \& Meus, P. (2004): Organic Contaminants in F. Zwahlen, (ed): Vulnerability and risk mapping for the protection of carbonate (karst) aquifers, final report (COST action 620). Pub. by: European Commission, Directorate XII Sci., Res. Dev., Rep., EUR 20912, Brussels.

Lekwot, V.E., Caleb, A.I. \& Ndahi, A.K. (2012) :Effects of effluent discharge of Kaduna Refinery on the water quality of River Romi.J. Res. Environ. Sci. Toxicol., 1(3):41-46.

Lovanh, N., Zhang, Y.K., Heathcote, R.C \& Alvarez, P.J. (200o): Guidelines to determine site-specific parameters for modelling the fate and transport of mono-aromatic hydrocarbons in groundwater. Submitted to: Iowa Comprehensive Petroleum Underground Storage Tank Fund Board, Westown Parkway: AON Risk Services Inc.

Mohammed, S.A.S., Naik, M., Fakruddin, S.P., \& Nazeer, Z.A.M. (2008): Studies of contaminant transport at an industrial waste dumpsite of Bangalore, India. Ambi-Agua, 3(3):55-66.

Raja, G. \& Venkatesan, P. (2010): Assessment of groundwater pollution and its impact in and around Punnam area of Karur District, Tamilnadu, India, E-J. Chem., 7(2):473-478..

Saskatchewan Ministry of Environment (2008) Risk-based corrective actions for petroleum hydrocarbon impacted sites. EPB 344, Canada..

Singh, C.K., Shashtri, S., \& Mukherjee, S. (2010): Integrating multivariate statistical analysis with GIS for geochemical assessment of groundwater quality in Shiwaliks of Punjab, India. Environ. Earth Sci., 62( 7):1387-1405.

Vivien, C., Caleb, A.I. \& Lekwot, V.E. (2012): Public health effects of effluent discharge of Kaduna refinery into River Romi, Greener J. Med.Sci., 2(3):64-69.

Williams, S.D., Ladd, D.E. \& Farmer, J.J. (2006): Fate and transport of petroleum hydrocarbons in soil and groundwater at Big South Fork National River and Recreation Area, Tennessee and Kentucky, 2002-2003, United State Geological Survey Scientific Investigations Report 2005-5104, 29p.

Yerima, F.A.K., Daura, M.M. \& Gambo, B.A. (2008): Assessment of Groundwater Quality in Bama Town, Nigeria. J. Sustain. Dev. Agricul. Environ., 3(2):128-137.

Yidana, S.M., Yakubo, B.B., \& Akabzaa, T.M. (2010): Analysis of groundwater quality using multivariate and spatial analyses in the Keta basin, Ghana, J. Afr. Earth Sci., 58(2):220-234.

Zaporozec, A . (2002) Groundwater contamination inventory, A methodological guide, IHP-VI Series on Groundwater No.2,Pub. by: UNESCO.161 p. 